\title{
Strict Identity with No Overlap
}

\author{
Achille C. Varzi \\ Department of Philosophy, Columbia University (New York)
}

(Final version published in Studia Logica 83 (2006): 371-378.)

It is natural to think that a standard, Kripke-style semantics for quantified modal logic (QML) is incompatible with the view that no individual can exist in more than one possible world, a view that seems to require a Lewis-style, counterpart-theoretic semantics instead. Strictly speaking, however, this thought is wrong-headed. A standard semantics regards a modal statement such as 'I might have been fat' as true only if I am in the extension of 'is fat' at some other possible world, whereas counterpart theory regards it as true only if a counterpart of mine is in the extension of 'is fat'. But just as the truth conditions of counterpart theory are in principle compatible with the possibility (rejected by Lewis) that some individuals qualify as their own other-wordly counterparts, the truth conditions of a standard semantics are in principle compatible with the possibility (dismissed by Kripke) that all individuals are worldbound. Here is how.

1. Consider a standard way of setting up the semantics. This involves two tasks. First, we have to say what sorts of structures qualify as models of the language, and then we have to spell out a corresponding definition of truth for all formulas of the language. Concerning the first task, the basic idea is that a model $\mathscr{N}$ must specify the following four ingredients:

- a non-empty set $W$ of possible worlds;

- an accessibility relation $R$ on $W$;

- a function $D$ assigning to each world $w \in W$ a non-empty domain of individuals $D(w)$;

- a function $V$ assigning to each variable $x$ an individual $V(x)$ and to each $n$-ary predicate $P$ an extension $V(P)(w)$ for each $w \in W{ }^{1}$

\footnotetext{
${ }^{1}$ If the language contains names, $V$ will also assign values to each name; to keep things simple, however, I shall assume the non-modal vocabulary to be that of pure quantification theory.
} 
On this basis, the second task is cashed out in terms of a recursive definition of the conditions under which a formula $\phi$ is true at a world $w$ in a model $\mathscr{N}$, written $\stackrel{\stackrel{\prime \prime}{=}}{=}$. For instance, with the usual connectives and quantifiers the definition goes like this:

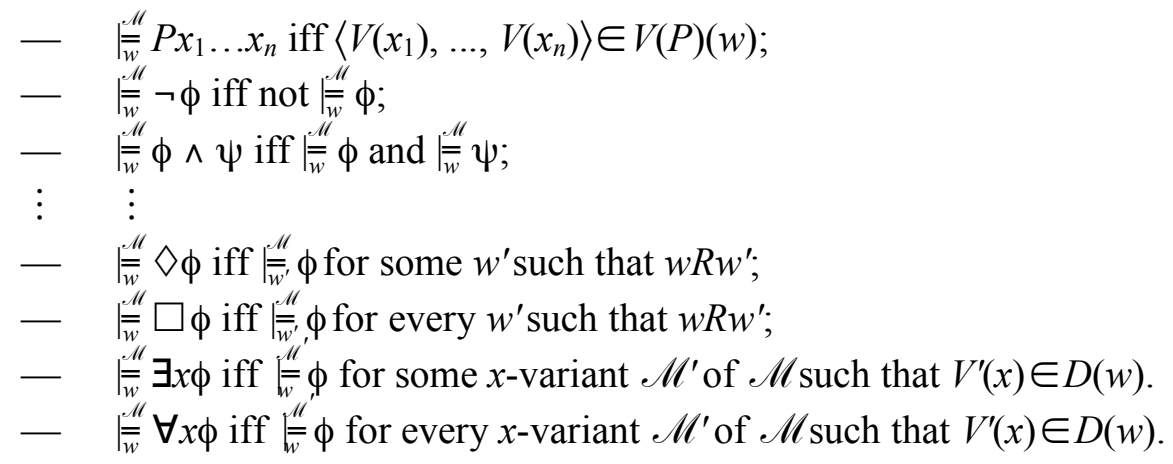

(where an $x$-variant of a model $\mathscr{M}=\langle W, R, D, V\rangle$ is any model $\mathscr{N}^{\prime}=\left\langle W^{\prime}, R^{\prime}\right.$, $\left.D^{\prime}, V^{\prime}\right\rangle$ that agrees with $\mathscr{M}$ on everything except possibly that $\left.V^{\prime}(x) \neq V(x)\right)$.

Of course, in order for this picture to qualify as a general semantic framework, it must be neutral with respect to what modal principles will qualify as valid. That is, it must only validate those formulas of QML that capture the logic of $\diamond$ and $\square$, as opposed to formulas that reflect controversial modal tenets. The distinction is not clear-cut, but it is intuitive enough to provide a criterion for testing the framework. For example, formulas such as

$$
\begin{array}{ll}
\text { K } & \square(\phi \rightarrow \psi) \rightarrow(\square \phi \rightarrow \square \psi) \\
\text { C } & (\square \forall x \phi \wedge \square \forall x \psi) \rightarrow \square \forall x(\phi \wedge \psi)
\end{array}
$$

are usually regarded as expressing logical truths, whereas propositional principles such as

$$
\begin{array}{ll}
\text { T } & \square \phi \rightarrow \phi \\
\mathbf{5} & \nabla \phi \rightarrow \square \diamond \phi
\end{array}
$$

or quantified principles such as the Barcan formula and its converse

$$
\begin{array}{ll}
\text { Bf } & \forall x \square \phi \rightarrow \square \forall x \phi \\
\text { Bf } & \square \forall x \phi \rightarrow \forall x \square \phi
\end{array}
$$

are not. Thus, in order for the picture to qualify as a general semantic framework, it must validate $\mathbf{K}$ and $\mathbf{C}$ but not the more controversial principles, though it should allow for the possibility that the latter be valid relative to certain classes of models. 
So a lot depends on how exactly we go about spelling out the details of the first task, i.e., the general definition of a model. Of the four ingredients that make up a model, the first ingredient - the set $W$-raises no issues (though one might have qualms concerning the requirement that it be non-empty), and the second - the relation $R$-raises issues that are not peculiar to QML: depending on what sort of relation we allow, the logical status of certain modal theses may vary. For example, if we require that $R$ be reflexive, then $\mathbf{T}$ will be valid, and if we require $R$ to be euclidean, then 5 will be valid. Yet in principle there is no constraint whatsoever on the available options, so we are free to allow for all the accessibility relations we wish. The other two ingredients, however, are peculiar of the semantics of QML and require that we make some further decisions. Specifically, we must decide (1) what sorts of conditions (if any) must be met by the domain function $D$, and (2) what sorts of conditions (if any) must govern the extension $V(P)(w)$ of a predicate $P$ at a world $w$.

Consider (1). Here the question is whether we have the same sort of flexibility that we have with regard to the accessibility relation. And the answer is twofold: On the one hand, (1.1) if we think that QML should be an extension of classical logic, then we have no flexibility whatsoever: unless all worlds are assigned exactly the same domain, some classically valid principles may fail to hold (for example, $\forall x \phi x \rightarrow \phi x$ may be false at a world $w$ where $V(x) \notin D(w)$ ). ${ }^{2}$ This requirement, however, has its costs. Among other things, such schemes as $\mathbf{B f}$ and $\mathbf{B} \mathbf{f}_{\mathbf{c}}$ would come out valid, along with the thesis of Necessary Existence:

$$
\text { NE } \forall x \square \exists y x=y,
$$

and most philosophers find such schemes unacceptable. On the other hand, (1.2) if we are willing to give up classical logic (and adopt, for example, a free logic), then we have all the flexibility we want: depending on what conditions we impose on $D$ (if any), the logical status of certain modal theses may vary, but there is in principle no constraint whatsoever on the available options.

As for (2), again we have two main options, depending on whether we do or do not think that the extension of a predicate at a given world may only in-

\footnotetext{
${ }^{2}$ One could stick to classical logic by disregarding such worlds as ineligible, i.e., by treating a formula $\phi$ as valid in a model $\mathscr{M}$ as long as it is true at every world in. $/$ whose domain contains a value for each variable free in $\phi$. (This is Hughes and Cresswell's strategy in their 1996, pp. 275-276.) However, in that case one would have to depart from the basic logic of $\square$ unless a different condition is imposed on $D$, namely that $D(w) \subseteq D\left(w^{\prime}\right)$ whenever $w R w^{\prime}$ (otherwise $\forall x \phi x \rightarrow \phi x$ would be valid but $\square(\forall x \phi x \rightarrow \phi x)$ would not), and such a condition is enough to validate $\mathbf{B f _ { c }}$.
} 
volve individuals from the domain of that world: (2.1) The first option amounts to the requirement that, for each $n$-ary predicate $P$ and each world $w$, the extension of $P$ at $w$ be a set $V(P)(w) \subseteq D(w)^{n}$. Such a requirement implies that an atomic formula $P x_{1} \ldots x_{n}$ can be true at a world $w$ only if each one of $V\left(x_{1}\right), \ldots$, $V\left(x_{n}\right)$ is an individual in $D(w)$, so the so-called thesis of Serious Actualism would be valid:

$$
\text { SA } \square\left(P x_{1} \ldots x_{n} \rightarrow \exists y x_{i}=y\right)
$$

This thesis, however, is philosophically controversial. Moreover, the resulting logic would be sui generis, as the relevant notion of validity would fail to be preserved upon substitution of atomic formulas by arbitrary formulas (for example, substituting $\neg P x_{1} \ldots x_{n}$ for $P x_{1} \ldots x_{n}$ in $\mathbf{S A}$ yields a non-valid formula). (2.2) The second option allows for the extension of a predicate $P$ at a world $w$ to be any relation $V(P)(w) \subseteq U^{n}$, where $U=\bigcup\{D(w): w \in W\}$ is the universe of all individuals in the model. This allows for greater flexibility, as it is entirely up to the model to fix the truth-value of any atomic sentence: no controversial principle is generally valid and the logic itself is not affected. So if we choose this option, we are then free to focus on the models we like best and figure out the logic that they determine.

2. Now, in his seminal 1963 paper, 'Semantical Considerations on Modal Logic', Kripke went for options (1.2) and (2.2) (see p. 86n) and most people have followed him on this score. The rationale for this choice is precisely that it allows for the greatest possible generality, though at the cost of some nonstandardness in the underlying extensional logic. Do you think the right propositional modal logic is S5? Then pick those models where $R$ is reflexive and euclidean. Do you believe in necessary existence? Then pick those models where $D$ is constant. In serious actualism? Then pick those models where $V$ interprets all predicates as subsets of the relevant domain of quantification. Such flexibility is most welcome indeed, if the semantics must be suitable to investigate the modal logic of different conceptions of how the world is and might have been. This is what makes Kripke-style semantics a tool rather than a theory. The only constraint that we have is that once we have made our choice and begin to engage in modal speculations, we must assume that Kripke was right about one controversial thing: that to speculate about whether a certain individual might have been so-and-so is to speculate about whether itthat very same individual - is so-and-so at some possible world. To evaluate a modal statement of the form $\diamond \phi x$ or $\square \phi x$ relative to a certain world $w$ is to evaluate the statement $\phi x$ at other worlds, changing the extension of the predi- 
cates occurring in $\phi$ but keeping the same value for $x$ throughout. Kripke-style semantics, at least in this form, embodies the view that one and the same individual may belong to more than one possible world. ${ }^{3}$

But now comes the point. To say that an individual may belong to more than one possible world is not to say that it must. Neither does it amount to saying that an individual $A$ can be numerically identical to, but qualitatively different from, an individual $B$. Precisely because of the great flexibility offered by the overall semantic framework set up by Kripke, among the many options that we have we are free to favor the following: Pick those models where all worlds have pairwise disjoint domains. That is, we are free to select as the only admissible models those structures $\mathscr{N}=\langle W, R, D, V\rangle$ that satisfy the following "no-overlap" requirement:

$$
\text { For all } w, w^{\prime} \in W \text {, if } w \neq w^{\prime} \text {, then } D(w) \cap D\left(w^{\prime}\right)=\varnothing .
$$

Does this contradict Kripke's views about identity? Surely not, for the view is trivially satisfied. Does it prevent us from engaging in modal talk? It does not, precisely because the framework does not require that the extensions of all predicates at a given world involve only individuals from that world. Consider, for example, a sentence of the form $\diamond P x$ and suppose we want to evaluate it at a world $w$. The model will assign to $x$ a value $V(x) \in D\left(w_{i}\right)$ for some world $w_{i}$. Perhaps the model also says that $w_{i}$ is not accessible from $w$. Or perhaps it says that $w_{i}$ is accessible but $V(x) \notin V(P)\left(w_{i}\right)$, in which case $P x$ would be false at $w_{i}$. Yet this is not to say that $\diamond P x$ must be false at $w$. By definition, $\diamond P x$ will be true at $w$ as long as $P x$ is true at some world $w^{\prime}$ accessible from $w$, regardless of whether $w^{\prime}=w_{i}$. In other words, $\diamond P x$ will be true at $w$ as long as there is some accessible world $w^{\prime}$ such that $V(x) \in V(P)\left(w^{\prime}\right)$, regardless of whether $V(x) \in$ $D\left(w^{\prime}\right)$. And surely there can be such worlds.

This might sound strange: how can the truth of our modal claim depend on whether $P x$ is true at some world in which $x$ does not exist? But the answer is built in the general apparatus via (1.2)-(2.2): at no world does the truth of $P x$ require that $x$ exists. One tends to ignore it when it comes to providing the in-

\footnotetext{
${ }^{3}$ Ersatzists (Lewis 1986: 136ff) might protest. Pace Kripke (1980: 45), one may take the domain of a possible world in Kripke-style semantics to be the set of individuals that that world represents as existing, without any implication that those individuals are literally speaking part of the world in question. Then let me rephrase the claim thus: Kripke-style semantics embodies the view that one and the same individual can be (represented as) existing at more than one possible world. In the following, however, I shall make no efforts to stick to this cumbersome terminology.
} 
tuitive rationale for the truth conditions of modal statements, but it is precisely for that reason that formulas such as

$$
\begin{array}{ll}
\text { A } & \diamond P x \rightarrow \diamond \exists x P x \\
\text { B } & \square P x \rightarrow \square \exists x P x
\end{array}
$$

are not generally valid in the semantics defined by (1.2)-(2.2). So if that is the general picture, then so be it also in the special case where all worlds are pairwise disjoint. The same applies to any statement of the form $\diamond \phi x$ and $\square \phi x$. Of course, sometimes it is crucial that $x$ exists precisely in that world at which it satisfies $\phi$. This is the case, for instance, when $\phi x$ says that $x$ exists, as in $\exists y x=y$. In general, however, we may embark on modal speculations about an individual by looking at what open formulas are satisfied by that individual in the accessible worlds, regardless of which of those worlds (if any) hosts the individual in question.

3. I can see various reasons why such an account might be found unpalatable. For one thing, I myself cannot put up with the thought that a predicate may be true of something that does not exist, and for that very reason-together with my sympathies for the no-overlap requirement - I do not accept the standard truth conditions for the modal operators. So I go counterpart theory. But that is beyond the point. Surely a serious actualist cum no-overlap has little use for a standard Kripke-style semantics. Yet the point is precisely that such semantics is not committed to serious actualism, and for that reason it is compatible with the no-overlap requirement.

Consider also this. I am not fat, but I could have been fat. So 'I am fat' is false at the actual world, $w$, but there is a possible world, $w$ ', at which 'I am fat' is true. That's the idea. As it turns out, however, this is not tantamount to saying that in such a world, $w^{\prime}$, I am any different from the way I am in $w$. In a nooverlap scenario, $w$ is the only world in which I exist, and I am what I am. Rather, $w^{\prime}$ should be thought of as a world in which, mirabile dictu, I fall into the extension of 'fat'. Now that's not quite what most of us have in mind when we engage in modal speculations. We don't care about how the extension of a predicate could change unless that change reflects a genuine change in the properties of the objects. More generally, we don't want an object to fall into the extension of a predicate ('fat') unless the object has the corresponding property (being fat). I agree. But, again, this is beyond the point. If nonexistent objects are allowed to fall into the extension of a predicate, this intuition is up for grabs. A standard example used to illustrate the non-seriousactualist intuition behind (2.2) is that a statement such as 'Pegasus is a mytho- 
logical creature' should count as true at the actual world, where Pegasus does not exist. Presumably, however, such a statement would count as false at a world where Pegasus exists. If so, then we already have a case in point: Pegasus - that beast - both would and would not have the one property of being mythological, depending on where the property gets ascribed to it. Whatever rationale lies behind (2.2), if one is happy with it in such cases one can hardly reject the no-overlap account - except on the basis of a substantive theory of properties.

So we may have metaphysical reasons to dislike a Kripke-style semantics cum no-overlap — we may be serious actualists, or we may think that only certain properties (e.g., being mythological, as opposed to being fat) may be enjoyed by non-existents. But then, again, we may equally have metaphysical reasons to dislike a Kripke-style semantics cum fixed domains, for instance. Such reasons matter a lot when it comes to the practice of philosophy, and we should be able to proceed as we see fit. Yet this is not to say that those semantics are inherently unintelligible.

Let us rather ask: are there any principles whose validity reflects the no-overlap requirement in a Kripke-style semantics? The answer is Yes-for example:

$$
\begin{array}{ll}
\text { NO }_{1} & (\diamond \phi x \wedge \diamond \neg \phi x) \rightarrow \diamond \neg \exists y x=y \\
\text { NO2 }_{2} & (\exists y x=y \wedge \phi x) \rightarrow \square(\exists y x=y \rightarrow \phi x) .
\end{array}
$$

If a formula and its negation can both be true, then they must be true at different worlds, and $x$ cannot exist in both unless their domain overlap. Likewise, if a formula is true at $a$ world where $x$ exists, then it is true at every world where $x$ exists. On the other hand, note that in spite of the no-overlap requirement the standard principles that assert the necessity of identity and the necessity of non-identity remain valid, though trivially so: ${ }^{4}$

$$
\begin{array}{ll}
\text { ID } & x=y \rightarrow \square x=y \\
\text { NI } & \neg x=y \rightarrow \square \neg x=y .
\end{array}
$$

It is worth remarking that none of these four principles would generally be valid on a counterpart-theoretic semantics for QML, so the above semantics is significantly different. But counterpart theory comes in two parts: the first is the metaphysical thesis that no individual exists in more than one world; the

\footnotetext{
${ }^{4}$ At least, these principles are valid if the equality predicate is assumed to stand for the identity relation defined on the whole universe $U$, as on most standard semantics.
} 
second is the semantic thesis that modal claims about a certain individual should be cashed out in terms of the individual's counterparts rather than in terms of the individual itself. It is certainly possible, though perhaps not so interesting, to accept only the semantic thesis, so as to rely on an individual's proper counterparts only in those worlds (if any) where the individual itself does not exist. The other option - to accept the metaphysical thesis while rejecting the semantic thesis-may be even less interesting, but it is equally viable.

\section{References}

Hughes, G. E., and Cresswell, M. J. (1996), A New Introduction to Modal Logic, London: Routledge.

Kripke, S. A. (1963), 'Semantical Considerations on Modal Logic', Acta Philosophica Fennica 16: 83-94.

Kripke, S. A. (1980), Naming and Necessity, Harvard University Press, Cambridge (MA).

Lewis, D. K. (1986), On the Plurality of Worlds, New York: Blackwell. 